\title{
Avaliação agronômica de genótipos de amendoim no estado de Goiás, na safra 2020/21
}

\author{
Submetido - 09 jul. 2021 \\ Aprovado - 31 jul. 2021 \\ Publicado - 30 set. 2021
}

\section{http://dx.doi.org/10.52755/sas.v.2i(edesp1)135}

Jair Heuert ${ }^{(D)}$

Programa de Melhoramento do Amendoim - Embrapa, Santo Antônio de Goiás, GO. E-mail: jair.heuert@embrapa.br.

Maxuel Fellipe Nunes Xavier (iD

Discente de Agronomia do IFMT Campus São Vicente - Centro de Referência de Campo Verde, Campo Verde, MT. E-mail: maxuelfellipe90@gmail.com.

Taís de Moraes Falleiro Suassuna ${ }^{D}$

Programa de Melhoramento do Amendoim - Embrapa, Santo Antônio de Goiás, GO. E-mail: tais.suassuna@embrapa.br.

\section{RESUMO}

O presente trabalho teve como objetivo a avaliação agronômica de linhagens de amendoim no estado de Goiás. O experimento foi realizado na área experimental da Embrapa Arroz e Feijão, localizado no município de Santo Antônio de Goiás-GO, no ano agrícola 2020/21, com semeadura manual realizada no dia 10 de dezembro de 2020. Foi utilizado delineamento em blocos casualizados, com quatro repetições. Os tratamentos constaram de nove genótipos, sendo oito linhagens (1968 OL, 2133 OL, 2259 OL, 2091 OL, 2010 OL, 1253 OL, 1973 OL e 1965 OL) e uma cultivar (BRS 421 OL), todas desenvolvidas pelo PMA da Embrapa. As parcelas foram compostas por duas linhas de três metros de comprimento, espaçamento ente linhas de $0,90 \mathrm{~m}$, intervalo entre parcelas de dois metros e parcela útil 5,4 $\mathrm{m}^{2}$. Os manejos cultural e fitossanitário foram realizados de acordo com as recomendações para a cultura. 0 estande médio foi de 14 plantas por metro linear. Foram avaliadas a severidade de mancha preta, a massa de 100 grãos e a produtividade de vagens. O genótipo BRS 421 OL obteve a menor nota de severidade de mancha preta, a maior massa de 100 grãos (granulometria) e encontra-se entre os genótipos de maior produtividade, juntamente com o 2259 OL, 2091 OL, 2010 OL e 1965 OL. Todos os genótipos avaliados apresentaram altos níveis produtivos, acima de 5.100 kg.ha-1, nas condições do estado de Goiás.

Palavras-chave: Arachis hypogaea L.; Programa de Melhoramento; Produtividade.

\section{Agronomic evaluation of peanut genotypes in the state of Goiás, 2020-21 crop season}

\section{ABSTRACT}

The aim of this study was the agronomic evaluation of peanut genotypes in the state of Goiás. The experiment was carried out in the experimental area of Embrapa Arroz e Feijão, Santo Antônio de Goiás-GO, 2020/21 crop season, under a randomized block design with four replications. The treatments consisted of nine genotypes, eight lines (1968 OL, 2133 OL, 2259 OL, 2091 OL, 2010 OL, 1253 OL, 1973 OL and 1965 OL) and one cultivar (BRS 421 OL), developed by the PMA from Embrapa. The plots consisted of two lines of three meters in length, spacing between lines of $0.90 \mathrm{~m}$, interval between plots of two meters and useful plot of $5.4 \mathrm{~m}^{2}$. Planting date: December 10, 2020. Crop management followed the regular recommendations for peanut in Brazil. The average stand was 14 plants per linear meter. The plots were inverted using a simple digger/inverter equipament; after the curing period in the

Este é um trabalho de acesso aberto e distribuído sob os Termos da Creative

Commons Attribution Attribution-NonCommercial-ShareAlike 4.0 International. 
field, the plots were harvested. The traits evaluated were late laef spot severity (LLSev), mass of 100 kernelsand pod yield. The cultivar BRS 421 OL had the lowest LLSsev the highest mass of 100 kernels (granulometry) and is among the genotypes with the highest productivity, along with 2259 OL, 2091 OL, 2010 OL and 1965 OL. All genotypes evaluated showed high production levels, above 5,100 kg.ha-1, in the conditions of the state of Goiás.

Keywords: Arachis hypogaea L.; Breeding Program; Yield.

Evaluación agronómica de genotipos de maní en el estado de Goiás

\section{RESUMEN}

El objetivo de este estudio fue la evaluación agronómica de genotipos de maní en el estado de Goiás. El experimento se llevó a cabo en el área experimental de Embrapa Arroz e Feijão, ubicada en el municipio de Santo Antônio de Goiás-GO, con siembra manual realizada el 10 de diciembre de 2020. Se utilizó un diseño de bloques al azar con cuatro repeticiones. Los tratamientos consistieron en nueve genotipos, ocho líneas (1968 OL, 2133 OL, 2259 OL, 2091 OL, 2010 OL, 1253 OL, 1973 OL y 1965 OL) y un cultivar (BRS 421 OL), todos todos desarrollados por la Embrapa. Las parcelas estaban compuestas por dos líneas de tres metros de longitud, espaciamiento entre líneas de $0,90 \mathrm{~m}$, intervalo entre parcelas de dos metros y parcela útil de $5,4 \mathrm{~m}^{2}$. El manejo cultural y fitosanitario se realizó de acuerdo con las recomendaciones para el cultivo. El rodal promedio fue de 14 plantas por metro lineal. La prueba se invirtió utilizando un simple iniciador y luego del período de secado en el campo, se cosecharon las parcelas. Las evaluaciones realizadas fueron severidad de mancha negra, masa de 100 granos y rendimiento de vaina. El cultivar BRS 421 OL tuvo la puntuación más baja de severidad de la mancha negra, la masa más alta de 100 granos (granulometría) y se encuentra entre los genotipos con mayor productividad, junto con 2259 OL, 2091 OL, 2010 OL y 1965 OL. Todos los genotipos evaluados mostraron altos niveles de producción, por encima de los 5.100 kg.ha-1, en las condiciones del estado de Goiás.

Palabras clave: Arachis hypogaea L.; Programa de Mejoramiento; Productividad.

\section{Introdução}

O amendoim (Arachis hypogaea L.) é cultivado em diversos países e amplamente utilizado na indústria de alimentos como uma importante fonte de proteína e óleo vegetal. Segundo o Departamento de Agricultura dos Estados Unidos (2021), a produção mundial de amendoim foi de aproximadamente 49,2 milhões de toneladas na safra 2020/21. Os maiores produtores mundiais dessa oleaginosa são a China (37,0\%), Índia (12,2\%), Nigéria (8,9\%), Estados Unidos (5,8\%), Sudão (5,1\%), Burma (3,3\%), Senegal $(3,2)$ e Argentina $(2,8 \%)$, sendo o Brasil o décimo segundo colocado com uma participação de 1,4\%. A área de amendoim plantada no mundo é de 28,80 milhões de hectares na safra atual. A produtividade média do Brasil é de 3,9 toneladas por hectare, ficando atrás somente dos Estados Unidos (4,5 toneladas por hectare) e da Argentina (3,7 toneladas por hectare). 
A cultivar BRS 421 OL possui o número de registro 37.301, na data de 31 de agosto de 2017 no Registro Nacional de Cultivares (RNC) do Ministério da Agricultura, Pecuária e Abastecimento (MAPA) (MAPA, 2021). Esta cultivar foi desenvolvida pelo Programa de Melhoramento de Amendoim (PMA) da mantenedora Empresa Brasileira de Pesquisa Agropecuária (EMBRAPA). É recomendada com descrição de adaptação ao bioma Cerrado no Brasil Central, compreendendo aos estados de Mato Grosso do Sul, Mato Grosso, Goiás e Tocantins (SUASSUNA et al., 2020).

Estudos são desenvolvidos com esta cultivar nos estados que possui adaptação: Mato Grosso do Sul (APARECIDO FILHO et al., 2019), Mato Grosso (SUASSUNA et al., 2018; SANTIN et al., 2019; RIZZI et al., 2019; XAVIER et al., 2020; SANTIN et al., 2020; RIZZI et al., 2020), Goiás (HEUERT et al., 2018; MARTINS et al., 2019; HEUERT et al., 2020) e Tocantins (SUASSUNA et al., 2018). Com base nos dados destes estudos, constata-se a provável adaptação desta cultivar a estes estados, com médias produtivas superiores à estimativa média nacional de amendoim primeira safra $\left(3.679,0 \mathrm{~kg}^{\mathrm{h}} \mathrm{ha}^{-1}\right)$ da CONAB (2021).

Por outro lado, o PMA desenvolve continuamente novas linhagens com características competitivas para o mercado de amendoim no Brasil, que requerem avaliação em diferentes locais e anos. As avaliações de linhagens de amendoim realizadas em Goiás são importantes para conhecer o desempenho das linhagens em condições de altitude moderada $(820 \mathrm{~m})$ e solo argiloso (mais de $50 \%$ de argila) e manejo ótimo da cultura. Com base nisso, o presente trabalho teve como objetivo a avaliação agronômica de genótipos de amendoim no estado de Goiás, na safra 2020/21.

\section{Material e métodos}

O experimento foi realizado na área experimental da Embrapa Arroz e Feijão, localizado no município de Santo Antônio de Goiás-GO, no ano agrícola 2020/21. O clima do município de Santo Antônio de Goiás-GO é do tipo Aw, ou seja, clima tropical com estação seca de inverno (KÖPPEN; GEIGER, 1928). A temperatura média do ar anual é de $23,1{ }^{\circ} \mathrm{C}$, 
apresentando período chuvoso de outubro a abril e período seco de maio a setembro. A umidade relativa média do ar anual é de $70 \%$ e a precipitação pluvial média anual é de 1.472,8 mm. O solo é predominantemente classificado como Latossolo Vermelho-Escuro, com textura argilosa e o relevo plano (SILVA et al., 2000).

Foi utilizado delineamento em blocos casualizados, com quatro repetições. Os tratamentos constaram de nove genótipos, sendo oito linhagens (1968 OL, 2133 OL, 2259 OL, 2091 OL, 2010 OL, 1253 OL, 1973 OL e 1965 OL) e uma cultivar (BRS 421 OL), todas desenvolvidas pelo PMA da Embrapa. As parcelas foram compostas por duas linhas de três metros de comprimento, espaçamento entre linhas de $0,90 \mathrm{~m}$, intervalo entre parcelas de dois metros e parcela útil $5,4 \mathrm{~m}^{2}$. A semeadura foi realizada manualmente, no dia 10 de dezembro de 2020.

Os manejos cultural e fitossanitário foram realizados de acordo com as recomendações para a cultura. Com base na análise química do solo da área experimental foi realizada uma adubação de semeadura de $200 \mathrm{~kg}$.ha-1 de Superfosfato Simples. Em cobertura foi feita aplicação de $500 \mathrm{~kg}$.ha-1 de gesso agrícola, aos 42 DAP. O estande médio foi de 14 plantas por metro linear. O ensaio foi invertido usando um arrancador simples da marca Agromérica $\AA^{\circledR}$ e após o período de secagem no campo as parcelas foram colhidas.

Foram avaliados severidade de mancha preta (notas), massa de 100 grãos (g) e produtividade de vagens $\left(\mathrm{kg}^{\mathrm{ha}} \mathrm{ha}^{-1}\right.$ e sacas.alqueire $\left.{ }^{-1}\right)$. A severidade de mancha preta (Cercosporidium personatum) foi avaliada usando a escala diagramática da incidência com notas de 1 a 9 ao final do ciclo dos genótipos, antes da inversão do experimento (SUBRAHMANYAM et al., 1982). Foram avaliados massa de 100 grãos (g) e produtividade de vagens (kg.ha-1 e sacas.alqueire ${ }^{-1}$ ), mediante a pesagem de vagens e grãos, da área de 5,4 $\mathrm{m}^{2}$ centrais das duas linhas da parcela.

Os dados obtidos foram submetidos à análise de variância (Teste $\mathrm{F}$ ) e as médias dos tratamentos foram comparados pelo teste de Scott-Knott a 
5\% de probabilidade, por meio do programa computacional SISVAR 5.6 (FERREIRA, 2019).

\section{Resultados e discussão}

O experimento apresentou diferença significativa para severidade de mancha preta (notas), massa de 100 grãos (g) e produtividade de vagens $\left(\mathrm{kg} \mathrm{ha}^{-1}\right)$ em função de diferentes genótipos de amendoim no estado de Goiás. A menor nota de severidade foi observada no genótipo BRS $421 \mathrm{OL}$ $(4,7)$, seguido dos genótipos 1968 OL $(6,8), 2133$ OL $(7,1), 2259$ OL $(7,1)$, 2010 OL $(7,2)$ e 2091 OL $(7,2)$. Por outro lado, tem-se o 1965 OL $(8,1)$ com a maior nota, seguido do 1973 OL $(7,7)$ e 1253 OL $(7,6)$ (Tabela 1).

Tabela 1. Severidade de mancha preta (notas), massa de 100 grãos (g) e produtividade de vagens ( $\mathrm{kg} \cdot \mathrm{ha}^{-1}$ e sacas.alqueire ${ }^{-1}$ ) em função de diferentes genótipos de amendoim no estado de Goiás. Santo Antônio de Goiás-GO, 2020/21.

\begin{tabular}{ccccc}
\hline \multirow{2}{*}{ Genótipos } & Severidade & Massa de 100 grãos & \multicolumn{2}{c}{ Produtividade de vagens } \\
\cline { 2 - 5 } & (notas) & $(\mathrm{g})$ & $\left(\mathrm{kg}^{-h a}{ }^{-1}\right)$ & (sacas.alqueire $\left.^{-1}\right)$ \\
\hline 2259 OL & $7,1 \mathrm{~b}$ & $72,6 \mathrm{e}$ & $6.453,9 \mathrm{a}$ & 624,8 \\
BRS 421 OL & $4,7 \mathrm{a}$ & $84,7 \mathrm{a}$ & $6.287,5 \mathrm{a}$ & 608,7 \\
$2091 \mathrm{OL}$ & $7,2 \mathrm{~b}$ & $73,1 \mathrm{e}$ & $6.048,4 \mathrm{a}$ & 585,5 \\
$2010 \mathrm{OL}$ & $7,2 \mathrm{~b}$ & $77,0 \mathrm{c}$ & $5.975,9 \mathrm{a}$ & 578,5 \\
$1965 \mathrm{OL}$ & $8,1 \mathrm{~d}$ & $75,3 \mathrm{~d}$ & $5.895,9 \mathrm{a}$ & 570,8 \\
$1253 \mathrm{OL}$ & $7,6 \mathrm{c}$ & $76,7 \mathrm{c}$ & $5.772,1 \mathrm{~b}$ & 558,8 \\
$1973 \mathrm{OL}$ & $7,7 \mathrm{c}$ & $77,9 \mathrm{~b}$ & $5.707,6 \mathrm{~b}$ & 552,5 \\
$2133 \mathrm{OL}$ & $7,1 \mathrm{~b}$ & $75,4 \mathrm{~d}$ & $5.597,8 \mathrm{~b}$ & 574,9 \\
1968 OL & $6,8 \mathrm{~b}$ & $73,4 \mathrm{e}$ & $5.189,7 \mathrm{~b}$ & 502,4 \\
\hline Média & 7,0 & 76,2 & $5.881,0$ & 573,0 \\
\hline C.V. (\%) & 3,6 & 0,9 & 6,3 & - \\
\hline Pr>Fc & $>0,0001^{*}$ & $>0,0001^{*}$ & $>0,0025^{*}$ & - \\
* - significativo a 5\% de probabilidade pelo teste F; médias seguidas pela mesma letra não \\
diferem entre si, pelo teste de Scott-Knott a 5\%; ns - não significativo; C.V. - coeficiente de \\
variação.
\end{tabular}

Os valores de severidade observados para os genótipos BRS 421 OL (6,8), 2133 OL $(7,2), 1973$ OL $(8,3)$ e 1253 OL $(8,2)$ foram semelhantes aos obtidos em avaliações conduzidas sob condições semelhantes de manejo e no mesmo local, na safra 2019/20, descritas por Heuert et al. (2020) e Martins et al. (2020). 
Além do BRS 421 OL apresentar a menor nota de severidade de mancha preta, o mesmo obteve maior massa de 100 grãos $(84,7 \mathrm{~g})$ e encontra-se entre os genótipos de maior produtividade, em comparação aos demais. No entanto, verifica-se as menores massas nos genótipos $2259 \mathrm{OL}$ (72,6 g), 2091 OL (73,1 g) e 1968 OL (73,4 g), sendo-as aproximadamente $14,5 \%$ inferiores a BRS 421 OL (Tabela 1). Valores semelhantes foram relatados por Martins et al. (2019), ao avaliar os genótipos BRS 421 OL (85,3 g), 1253 OL (75,4 g), 2133 OL (75,6 g) e 1968 OL (73,5 g).

As maiores produtividades de vagens foram observadas nos genótipos $2259 \mathrm{OL}$ (6.453,9 kg.ha-1), BRS 421 OL (6.287,5 kg.ha-1), $2091 \mathrm{OL}$ (6.048,4 kg.ha-1), 2010 OL (5.975,9 kg.ha-1) e 1965 OL (5.895,9 kg.ha-1). Os demais genótipos: 1253 OL (5.772,1 kg.ha-1), 1973 OL (5.707,6 kg.ha-1), 2133 OL $\left(5.597,8 \mathrm{~kg} \mathrm{ha}^{-1}\right)$ e 1968 OL $\left(5.189,7 \mathrm{~kg} \cdot \mathrm{ha}^{-1}\right)$, obtiveram produtividades inferiores em comparação aos demais e a média do experimento (5.881,0 kg.ha-1) (Tabela 1). Ressalta-se que o nível de produtividade do presente estudo foi alto, pois o genótipo menos produtivo (1968 OL, 5.189,7 kg.ha ${ }^{-1}$ ) possui incremento de $41 \%$ quando comparado à estimativa média nacional $\left(3.679,0 \mathrm{~kg} \cdot \mathrm{ha}^{-1}\right)$ da CONAB (2021).

\section{Conclusões}

O genótipo BRS 421 OL obteve a menor nota de severidade de mancha preta, a maior massa de 100 grãos (granulometria) e encontra-se entre os genótipos de maior produtividade, juntamente com o 2259 OL, 2091 OL, 2010 OL e 1965 OL. Sobretudo, todos os genótipos apresentaram altos níveis produtivos, acima de $5.100 \mathrm{~kg} \cdot \mathrm{ha}^{-1}$, nas condições do estado de Goiás.

\section{Agradecimentos}

Os autores agradecem ao projeto SEG 20.18.01.021.00 da Embrapa. O agradecimento também é estendido as empresas da cadeia produtiva do amendoim que aderiram ao Programa de Melhoramento do 
Amendoim (PMA), que fomentam a pesquisa científica e contribuem para o desenvolvimento de novas cultivares de amendoim. 


\section{Referências}

APARECIDO FILHO, A. C.; RODRIGUES, C. R.; HEUERT, J.; MARTINS, K. B. B.; SUASSUNA, T. M. F.; SOUZA, T. C. Desempenho de cultivares de amendoim nas condições do Mato Grosso do Sul. In: Anais do encontro sobre a cultura do amendoim, 16., 2019, Jaboticabal. Anais eletrônicos... Campinas, GALOÁ, 2019.

CONAB. Acompanhamento da Safra Brasileira de Grãos. Safra 2020/21 - Sétimo levantamento, v. 8, n. 7, p. 1-117, 2021. Disponível em: <https://www.conab.gov.br/info-agro/safras>. Acesso em: 04 jul. 2021.

DEPARTAMENTO DE AGRICULTURA DOS ESTADOS UNIDOS. World Agricultural Production. Foreigh Agricultural Service wap 6-21, 43 p. 2021. Disponível

em: $<$ https://apps.fas.usda.gov/psdonline/circulars/production.pdf>. Acesso em: 04 jul. 2021.

FERREIRA, D. F. SISVAR: um sistema de análise de computador para efeitos fixos projetos de tipo de partida dividida. Revista Brasileira de Biometria, v. 37, n. 4, p. 529-535, 2019.

HEUERT, J.; SUASSUNA, T. M. F.; MARTINS, K. B. B.; RIBEIRO, T. C. N.; GONÇALVES, W. C. Desempenho agronômico de genótipos de amendoim no estado de Goiás. In: Anais do encontro sobre a cultura do amendoim, 15., 2018, Jaboticabal. Anais eletrônicos... Campinas, GALOÁ, 2018.

HEUERT, J.; RODRIGUES, L. L.; MARTINS, K. B. B.; XAVIER, M. F. N.; SUASSUNA, T. M. F. Desempenho agronômico de novas linhagens de amendoim no Cerrado. South American Sciences, v. 1, n. 1, p. e2008, 2020.

KÖPPEN, W.; GEIGER, R. Klimate der Erde. Gotha: Verlagcondicionadas. Justus Perthes. 1928.

MAPA. CultivarWeb: BRS 421 OL. 2021. Disponível em: $<$ https://sistemas.agricultura.gov.br/snpc/cultivarweb/detalhe_cultivar.php? codsr $=37262>$. Acesso em: 04 jul. 2021.

MARTINS, K. B. B.; RODRIGUES, L. L.; HEUERT, J.; XAVIER, M. F. N.; SUASSUNA, T. M. F.; BETIOL, R. A. B. Desempenho agronômico de novas linhagens de amendoim no Cerrado. In: Encontro Sobre a Cultura do Amendoim, 16., 2019, Jaboticabal. Anais eletrônicos... Campinas: GALOÁ.

MARTINS, K. B. B.; HEUERT, J.; RODRIGUES, L. L.; XAVIER, M. F. N.; SUASSUNA, T. M. F. Desempenho agronômico de genótipos de amendoim tipo runner, visando colheita antecipada. South American Sciences, v. 1, n. 1, p. e2007, 2020. 
RIZZI, T. S.; OLIBONE, D.; LODEA, L.; HEUERT, J.; XAVIER, M. F. N.; SUASSUNA, T. M. F. Desempenho de cultivares de amendoim na região Médio-norte Mato-Grossense. In: Anais do encontro sobre a cultura do amendoim, 16., 2019, Jaboticabal. Anais eletrônicos... Campinas, GALOÁ, 2019.

RIZZI, T. S.; OLIBONE D.; HEUERT, J.; XAVIER, M. F. N.; MOURA, G. M.; SUASSUNA, T. M. F. Desempenho agronômico de genótipos de amendoim nas condições de Sorriso-MT. South American Sciences, v. 1, n. 2, p. e2043, 2020.

SANTIN, V.; PEROZINI, A. C.; ARAÚJO, C.; GIRON, F. G.; HEUERT, J.; XAVIER, M. F. N.; SUASSUNA, T. M. F. Desempenho de cultivares de amendoim nas condições de Campo Verde-MT. In: Anais do encontro sobre a cultura do amendoim, 16., 2019, Jaboticabal. Anais eletrônicos... Campinas, GALOÁ, 2019.

SANTIN, V.; HEUERT, J.; XAVIER, M. F. N.; RODRIGUES, M. A.; SUASSUNA, T. M. F. Desempenho agronômico de linhagens finais de amendoim nas condições de Campo Verde-MT. South American Sciences, v. 1, n. 1, p. e2011, 2020.

SILVA, S. C.; XAVIER, L. S.; PELEGRINI, J. C.; DAVID, F. A. Informações meteorológicas para pesquisa e planejamento agrícola 1999. Santo Antônio de Goiás: EMBRAPA Arroz e Feijão, 2000. 30 p.

SUASSUNA, T. M. F. et al. 'BRS 421' and 'BRS 423': high oleic peanut cultivars for production in Brazil. Crop Breeding Applied Biotechnology, v. 20, n. 1, p. e28932018, 2020.

SUASSUNA, T. M. F.; HEUERT, J.; BOGGIANI, J. C.; PERINA, F. J.; SOFIATTI, V.; BETTINI, P. C.; OLIVEIRA, M. C. T.; LEONEL, C. L. Desempenho de linhagens de amendoim na região do Cerrado. In: Anais do encontro sobre a cultura do amendoim, 15., 2018, Jaboticabal. Anais eletrônicos... Campinas, GALOÁ, 2018.

SUBRAHMANYAM, P.; MCDONALD, D.; GIBBONS, R. W.; NIGAM, S. N.; NEVILL, D. J. Resistance to rust and late leaf spot diseases in some genotypes of Arachis hypogaea. Peanut Science, v. 9, p. 9-14, 1982.

XAVIER, M. F. N.; MEINKE, G. C.; HEUERT, J.; MARTINS, K. B. B.; SUASSUNA, T. M. F. Desempenho agronômico de genótipos de amendoim nas condições de Campo Verde-MT. South American Sciences, v. 1, n. 1, p. e2009, 2020. 